\title{
An Improved Approach to Line Balancing for Garment Manufacturing
}

\author{
A. N. Wickramasekara \\ University of Sri Jayewardenepura, Sri Lanka \\ H. S. C. Perera \\ Sri Lanka Institute of Information Technology, Sri Lanka
}

\begin{abstract}
Production managers in the apparel industry frequently face the issue of being unable to complete the orders at the scheduled time. One of the reasons for this issue is the unavailability of a Line Balancing procedure that could encompass the stochastic nature of the sewing process, which is manifested through the likes of variability of sewing times, machine breakdowns, correcting defective products, and operator breakings such as for changing bobbins and drinking water. The objective of this research is to introduce a diverse approach to Line Balancing through giving due consideration to the stochastic nature of the process. The improved approach was developed through case study approach. Having selected a sewing line in an apparel factory, the process times of operations, major random events and the times elapsed between random events were recorded. Then the whole production cycle was simulated using ARENA software. By attempting and analysing different scenarios, a different approach for line balancing was introduced. The initial steps of the algorithm developed includes: collecting processing times and necessary information on manufacturing process, fitting standard probability distributions to both value added and non-value added activities, developing the precedence diagram, developing an initial algorithm for balancing a production line
\end{abstract}

\section{Corresponding Author:}

A. N. Wickramasekara is a Senior Lecturer at the Department of Decision Sciences, University of Sri Jayewardenepura, Nugegoda, Sri Lanka. E-mail: amila@sjp.ac.lk 
and finally, making necessary adjustments to the algorithm analyzing different scenarios. In order to check the validity of the algorithm, a production line was balanced for two different daily production targets. Thus, the application of proposed algorithm to balance the production line reduces the gap between the expected production target and the real achievement.

\section{Keywords}

Apparel Industry, Garment Factory, Line Balancing, Simulation

\section{Introduction}

Garment is one of the most important products for human beings. Garment industry has traditionally been the first step towards industrialization in developing countries. The Sri Lankan garment industry experienced a massive growth in last four decades and continues to be the strongest manufacturing sub-sector. Since Sri Lanka is a labour-surplus economy, growth of garment industry in past few years has generated large quantities of manufacturing employments, particularly for women. Garment factories in Sri Lanka face competition from other developing countries of South and South East Asia, such as India, Bangladesh, Pakistan, Indonesia, Cambodia, Laos and Vietnam. China also has emerged as a dominant force in the global apparel industry with its massive supply capability and very low costs of production. One of the most significant factors affecting the competitiveness of the Sri Lankan garment industry is low productivity. Sri Lankan factories' main competitive dimension is quality. During the past decade, different kinds of productivity improvement projects were implemented in Sri Lankan factories with a view to reduce the production cost while maintaining the high quality. According to the Sri Lanka Institute of Textile and Apparel, garment factories in Sri Lanka have been categorized as small and medium scale, large scale and extra-large scale based on number of sewing machines in the factory. The main process of converting raw materials in to a garment is common. But, according to the experience of the institute, the types of problems faced by garment factories are dependent on the scale of factories in Sri Lanka. Number of methods has been introduced by researchers to improve productivity in organizations. Some techniques deal with specific problems such as inventory, quality and set-up time. Although a garment factory consists of several departments, 
there is no doubt that the sewing department is the most important department in the whole firm. Because there are a lot of different operations which are done manually, the sewing department has to be under constant control. Improving the productivity in manufacturing significantly affects the overall efficiency in an organization. Line Balancing is one of the techniques that can be used to enhance the productivity in production lines. Job assignment to operators in a production line by means of a scientific approach is extremely important to maintain the consistency of operating efficiency. This research focuses on problems confronted by garment factories due to labour and resource allocation practices they follow. Different kinds of Line Balancing algorithms have been applied for many years in apparel industry for job assignments and resource allocation. However, manual-operations oriented system of this sector makes it impossible to gain certain results with the algorithms currently used. A lot of factors cause variations on operational time of the same task such as the stochastic nature of operations, the experience of the operator, quality of the environment and performance of the machinery. Such variations on task time cause the Line Balancing problem in the clothing industry to become more complicated. Moreover, other major stochastic variables such as machine breakdown, re-working, absenteeism, the work of the supervisor, maintenance etc. significantly affect to imbalance a production line. Therefore, managers are up against unexpected bottlenecks, increasing idle time, decreasing level of efficiency, increasing operator fatigue and increasing the defect rate during the sewing process. As a result of that, production managers are unable to complete the orders at the scheduled time. One of the main reasons for above mentioned difficulties is unavailability of a Line Balancing procedure that could encompass the stochastic nature of the garment manufacturing process, which is manifested through the likes of variability of operating times, machine breakdowns, reworking, and operator breakings.

Therefore, the objective of this research is to introduce a new Line Balancing algorithm through giving due consideration to the stochastic nature of the garment manufacturing process. When considering the variability of operating times, it is required to build up a new mechanism to model it. Presently, there are many garment factories in Sri Lanka. Out of them, a large scale garment factory located in Colombo district was selected 
for this study. Although the selected factory has 43 production lines, this research was conducted based on a single production line which operates at the highest efficiency level with minimum inconsistencies.

\section{Literature Review}

An assembly line is defined (Baybars, 1986, p. 909) as a set of distinct tasks which is assigned to a set of workstations linked together by a transport mechanism under detailed assembling sequences specifying how the assembling process flows from one station to another. A task is a smallest indivisible work element, and the order in which the tasks can be performed is restricted by a set of precedence relationships. The time required for the completion of a task is known as the task time (process time). The cycle time (station time) is the amount of time available at each station as well as the time between successive units coming off the line. Generally, the cycle time is predetermined based on the demand for the product in the given period (and/or the given operating time for the manufacturing system in that period), in other words, by what rate of production is desired. Line balancing is classified into the following categories according to the work of Bhattacharjee and Sahu (1987, pp. 32-43):

Single model line: deterministic task time. It is assumed that the task time of work element in each station is constant and a unique product is produced in each line.

Single model line: stochastic task time. Same as (1) but the station times are assumed to be independently normally distributed with known mean and variance.

Multi/mixed model line: deterministic task time. It is assumed that the task time of work element in each station is constant and an assembly line produces more than one style of the same product.

Multi/mixed model line: stochastic task time. Same as (3) but the station times are assumed to be independently normally distributed with known mean and variance.

The assembly line balancing problem deals with the amount of work, in terms of time, which has to be done at each workstation, given the 
precedence requirements. In the context of the assembly line balancing problem, the workload allocation problem in production lines is analogous, where the overall constraint is that the sum of the expected services times is a fixed constant and the allocation problem is basically to allocate the total time among the workstations, so as to optimize a given objective function usually throughput or average work-in-process (Papadopoulos, O'Kelly, Vidalis \& Spinellis, 2009). In the assembly line balancing problem there are three givens: a table of work elements with their associated times; a precedence diagram showing the element precedence relationship; and required output rate from the line. It is required to determine: the number of workstations; the number of operations at each workstation; and the work elements to be done in each workstation. The purpose of line balancing is to minimize total idle time (Konz \& Johnson, 2004). The traditional methods of assembly line balancing assume that the operation times at each station are fixed or deterministic. This assumption is not realistic since often these times are random variables (Nkasu \& Leung, 1995).

Different types of Line Balancing procedures have been introduced for the clothing industry to operate production lines at higher productivity level. Balancing is the technique of maintaining the same level of inventory at each and every operation at any point of time to meet the production target. Thus, this technique enables us to balance the work load of each operation to make sure that the flow of work is smooth, thus bottlenecks are not created.

\section{Widely Applied Balancing Procedure}

This procedure defined in work study is the most popular one in the Sri Lankan apparel sector. Steps of this method can be listed as follows. (International Labour Office, Introduction to Work Study)

1. Collect the necessary information required; the list of operations in sequence, the standard time for each operation, the length of the working day and the planned output rate.

2. Compute the capacity per hour for each operation

3. Determine the required output rate

4. Workout the required Theoretical Manning Level for each operation to maintain the required output rate. 
5. When you have fraction of operators, combine those operations with similar equipment to get operators with full numbers.

6. Assign operators to perform each operation considering the above calculation and the skill level of operators.

Standard time is the total time in which a job should be completed at the standard performance. The unit that measures the amount of work to be done by an operator in an operation by the number of minutes it should be completed in. Shaumon, Zaman and Rahman (2010) have shown the capability of above mentioned procedure to enhance the productivity significantly. To perform necessary calculations, following formulas have been used.

Theoretical Manning Level $=\frac{\text { Target Per Hour }}{\text { Process Capacity Per Hour }}$

Labour Productivity $=\frac{\text { Total Number of Output Per Day }}{\text { Number of Workers Worked }}$

Line Efficiency $=\frac{\text { Total Output Per Day x SAM }}{\text { Total Manpower Per Line } \mathrm{x} \text { Total Working Minutes Per Day }} \times 100 \%$

\section{Line Balancing using Simulation Techniques}

Although the apparel manufacturing process consists of large number of stochastic variables with complex relationship, deterministic models so far developed fail to reflect the scenario. Simulation is one of the most effective techniques that can be used to model the stochastic nature in the apparel manufacturing process. Several researchers have studied assembly line performance by using simulation techniques.

Güner and Ünal (2008) balanced a T-shirt manufacturing process using simulation techniques in which ARENA 7.0 simulation software has been used. Assumptions in this model are as follows.

- The assembly line is never starved.

- Set-up times are not taken into consideration.

- No maintenance processes are performed during the working period.

- All types of breakdowns are insignificant. 
- Transportation of raw materials is performed by workers who are not engaged in sewing operations.

After recording time duration to perform each operation, ARENA Input Analyser has been used to determine the most appropriate distribution for each operation. Then, the simulation model has been constructed and validated using the two-independent sample t-test. After validation process, different alternative models have been developed. Based on the queue length and the utilization of each resource, the best model has been obtained.

Kursun and Kalaoglu (2009) considered the random variability of operations to develop a simulation model for a shirt manufacturing process.Firstly, detailed work and time studies have been performed along the line. Secondly, to set up a model of the line by simulation, real data taken from a factory floor has been tested for distribution fit and a Kolmogrov-Smirnov test has been conducted for goodness of fit. Then, the data gathered has been transformed into a simulation model. After verification of the model by comparing it with the actual system, bottlenecks in the production line have been determined and possible scenarios have been tried by various what-if analyses to eliminate the bottlenecks and to suggest decision alternatives to manufacturers. To set up the model, an Enterprise Dynamics simulation program has been used. In this study, researchers have used three performance measures to compare alternatives; the average stay times of jobs in queues, the average content of jobs in queues and the average daily output.

Although number of methods was used to balance a production line, the reality is not presented. Even though, algorithms have been developed in deterministic approaches, the relationships between different types of stochastic variables are not taken into consideration. In most recent researches, simulation has been used as a powerful tool to balance a production line. In simulation approaches, different types of configurations have been made through what-if analysis observing bottleneck operations. But, no algorithm has been developed so far using simulation approach to balance a production line. 


\section{Methodology}

When considering both value added and non-value added operations, informants are the operators attached to the selected production line. The interested variable is the processing time for different activities performed by operators. All recorded processing times are primary data which were gathered through direct personal observations. When modelling a production line which is running under standard conditions, it is extremely important to use the data which are collected under the standard operating environment. Therefore, a production line utilizing with highly experienced operators was selected for this study. Almost all operators in the line were well familiar with respective operations because the order is being running for a long period of time. To simulate a production line, a large number of data should be collected in recognizing patterns of operations. Not only the valued added operations but also the major non-value added operations such as separating, counting of semi-finished garments and bundle handling were taken in to consideration when collecting data. Although, 20 operators were utilized in the production line, only 10 operators were considered in the data collection process considering their standard working procedure. The data collection process was conducted under two phases. During the first phase, cycle times of operations were collected along the production line. Processing times corresponding to non-value added activities were recorded during the second phase.

The process time is the interested variable in recording data. The purpose of gathering process times is fitting the most appropriate probability distribution for each activity. Therefore, more than 50 observations were made from selected operators with regard to their cycle times and considered non-value added activities. Out of them, only consistent processing times were selected for the analysis.

A number of quantitative techniques were applied for the analysis. For the statistical analysis, SPSS software was used. Firstly, processing times gathered under the standard operating environment were selected by removing the data with inconsistencies. Secondly, probability distributions were fitted for each operator's different kinds of activities using ARENA Input Analyser. After that, an initial algorithm was developed in order to balance a production line. Next, work of each operator was modelled and it 
was run according to the steps of the algorithm. Simulated daily production output was used throughout the algorithm in decision making process. By changing different parameters of the production process, the initial algorithm was finalized.

\section{Data Analysis and Results}

The data collection process was started by collecting the cycle times of value added operations from each operator. The main objective of recording cycle times is fitting the most appropriate probability distributions. According to the statistical theories, at least 30 observations without causing inconsistencies should be used to obtain accurate estimation. Furthermore, accuracy can be increased by taking large samples. So, the investigator conducted the data collection process with a view to obtaining 50 cycle times without inconsistencies. But, processing times without inconsistencies are infrequent in the real production environment. Hence, each and every operator was studied until 50 cycle times without inconsistencies were observed. After recording cycle times, non-value added operations were taken in to consideration to collect data. Three types of major non-value added activities could be observed in the production line: separating garments, counting garments and bundle handling. However, separating of garment is not necessary to be performed by all operators because it depends on the nature of the production process. Counting should be done by every operator to assure the right quantity of the bag before sending it to the next operator. But, majority of operators don't do it as a practice. Some operators rarely count garments. The bundle handling is a compulsory activity performed by every operator. In the real production line, nonproductive activities are highly affected by large number of inconsistencies. Therefore, those activities which are performed by every operator were not taken in to consideration to fit probability distributions.

The summary of fitted probability distribution for all value-added and non-value added activities can be displayed in the table 1.These distributions will be used to model the production line for simulation. After fitting the probability distributions, Kolmogorov Smirnov test was applied in order to validate them. Test was performed at 5percent level of significance. According to the results all fitted probability distributions were validated using ARENA input analyser. 
Table 1: Fitted probability distributions

\begin{tabular}{|c|c|c|}
\hline \multicolumn{2}{|r|}{ Operation } & Distribution \\
\hline \multirow{9}{*}{$\begin{array}{l}\text { Value Added } \\
\text { Operations }\end{array}$} & Pre-fix centre front over lap & $10+3 *$ Beta $(1.17,2.38)$ \\
\hline & Two needle join side seam & $13+\operatorname{Gamma}(0.953,2.05)$ \\
\hline & Zig-Zag binding under arm & $11.2+$ Weibull $(4.41,3.01)$ \\
\hline & $\begin{array}{l}2 \text { needle flat seam elastic cup neck } \\
\text { edge }\end{array}$ & $\begin{array}{l}6.05+1.88 * \text { Beta }(1.16, \\
1.19)\end{array}$ \\
\hline & $\begin{array}{l}2 \text { needle flat seam elastic attach } \\
\text { under band }\end{array}$ & $\begin{array}{l}9.61+\text { Lognormal }(1.46, \\
0.851)\end{array}$ \\
\hline & $\begin{array}{l}3 \text { step zigzag elastic attach back } \\
\text { appex }\end{array}$ & $17+\operatorname{Beta}(1.64,2.16)$ \\
\hline & Bar Tack at front apex edge & $14+4.87 *$ Beta $(1.13,1.7)$ \\
\hline & Hook and eye insert \& care label & Triangular $(30.5,32.4,35)$ \\
\hline & Trimming & $23+7 *$ Beta $(1.54,1.47)$ \\
\hline \multirow{3}{*}{$\begin{array}{c}\text { Non } \\
\text { Value Added } \\
\text { Operations }\end{array}$} & Separating 48 garments & $\begin{array}{l}138+81 * \text { Beta }(0.743, \\
1.09)\end{array}$ \\
\hline & Counting 48 garments & $27+10 *$ Beta $(1.53,1.49)$ \\
\hline & Bundle Handling & Normal $(73.1,7.42)$ \\
\hline
\end{tabular}

\section{Proposed Algorithm}

In this algorithm, " $i$ " is used as a variable to represent the workstation number. The smallest value which can be assumed for " $i$ " is one and it is the initial value at the starting point. " $i=1$ " represents the first workstation and any positive integers can be assumed for "i". " $\mathrm{m}$ " is a parameter used in this algorithm, which represents the maximum number of feasible operations that can be added to a workstation. Above analysis has been performed assuming $m=2$. It says that the maximum number of operations that can be added to one workstation is two. "m" can assume any positive integer. In this algorithm, " $\Theta$ " is used to represent the proportion of simulated daily production quantity which is greater than the daily production target to be achieved. " $\Theta_{0}$ " is a specific value of $\Theta$ that should be decided by a responsible officer for the production. To demonstrate this algorithm 0.95 was assumed for $\Theta_{0}$. In other words, If 95 percentof the simulated daily production quantity exceeds the daily production target to be achieved, it can be decided that the workstation has enough capacity to achieve the pre- 
defined required daily production target and the workstation has the extra capacity to perform more operations. The graphical representation of the algorithm is depicted in the figure 1.

Figure 1: Proposed algorithm

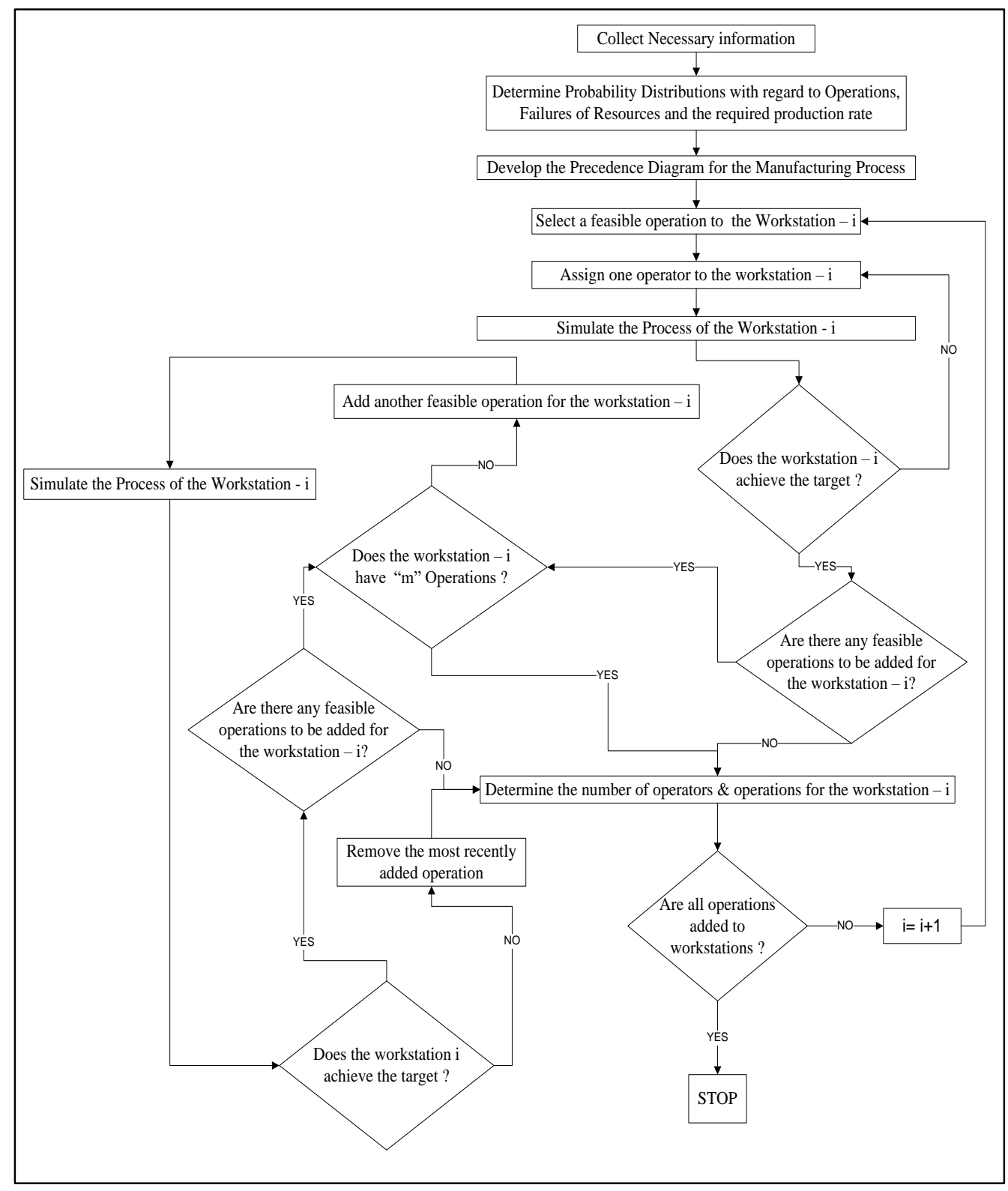


In order to show the applicability of the above explained line balancing algorithm, determination of number of operators and operations for workstations will be explained considering two cases. In the case 1, the daily production target is assumed as 2400 garments while in the case 2, it is 450. In the decision making process, it was assumed that every operation which is defined in the production process can be performed by any operator.

Figure 2: Precedence diagram

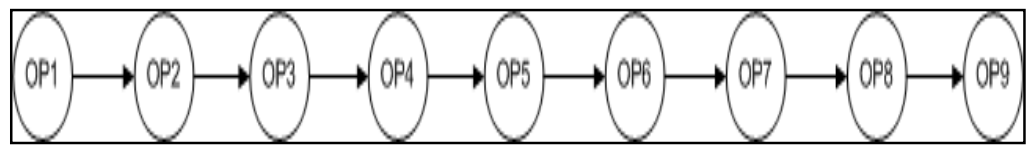

\section{Case I - Balancing the production line for 2400 garments per day Determination of the first workstation}

To begin with, a feasible operation and one operator were added to the first workstation. Then, the work of the first workstation was simulated and at the end of the run, simulated daily production output values of the workstation were analysed by calculating and analysing the $\Theta$ value. Calculated value of $\Theta$ was 1 which was greater than the pre specified value of $\Theta_{0}$. It is 0.95 for this demonstration. Therefore, it was concluded that the daily production output of the first workstation is achieved 2400 garments per day. Accordingly, it was decided that the first workstation has the extra capacity to perform additional operations. According to the precedence diagram (figure 2), there are feasible operations which can be added to the first workstation. In this balancing process, more than two operations are not added to a single workstation. But, only one operation has been added to the first workstation so far. Therefore, another feasible operation was added to the first workstation in addition to the previous operation. After that, the work of the first workstation was simulated and daily output values were analysed by using $\Theta$. The calculated value for the $\Theta$ is 0.65 which is less than 0.95 . Therefore, it was concluded that the required daily production target for two feasible operations cannot be achieved by one operator. Consequently, the most recently added operation to the first workstation was removed according to the algorithm. Finally, it was concluded that one operator is adequate for the first workstation and he should perform only the first feasible operation in order to achieve the daily production target. 


\section{Determination of the second workstation}

After making all the decisions with regard to the first workstation, a feasible operation and a new operator were added to the second workstation. Then, the simulation was started for the work of the first workstation and the second workstation. At the end of the run, simulated daily production output of the second workstation was analysed by using $\Theta$. The calculated value of $\Theta$ is 0.57 . Therefore, it can be concluded that the daily production target is not achieved by the operator assigned to the second workstation. Accordingly, it was decided that the second workstation does not have enough capacity to perform the assigned operation. Therefore, another operator was added to the second workstation. After that, the work of the first two workstations was simulated. Next, the output of the second workstation was analysed by using $\Theta$. Calculated value of $\Theta$ based on the output was 1 . Therefore, it was concluded that the first workstation and the second workstation are in a position to achieve 2400 garments per day. Hence, it was decided that the second workstation has the extra capacity to perform additional operations. Then, availability of feasible operations for the workstation was concerned. But, more than two operations cannot be assigned to one workstation according to pre-defined specifications. But, only one operation was assigned to the second workstation so far. Therefore, another feasible operation was added to the second workstation in addition to the previous operation. After that, the work of both workstations was simulated and the daily production output of the second workstation was analysed. Again, the value of $\Theta$ was calculated. It was 0.49 . Therefore, it was concluded that the second workstation with two operations cannot be run by one operator in order to achieve 2400 garments per day. Therefore, the most recently added operation to the second workstation was removed. Consequently, it was concluded that two operators are adequate to perform the assigned feasible operation for the second workstation to achieve the required target. Above procedure was applied to determine the number of operations, feasible operations to workstations and number of workstations for the production line assuming the daily production target of 2400 garments. The result of the analysis is shown in the table 2. As seen in the table 2, 19 operators are required to achieve the daily production target which is 2400 garments per day. 
Table 2: Work allocation for 2400 garments per day

\begin{tabular}{|c|c|l|}
\hline Workstation & $\begin{array}{c}\text { Number of } \\
\text { Operators }\end{array}$ & Operation \\
\hline 1 & 1 & Pre-fix centre front over lap \\
\hline 2 & 2 & Two needle join side seam \\
\hline 3 & 2 & Zig-Zag binding under arm \\
\hline 4 & 2 & 2 needle flat seam elastic cup neck edge \\
\hline 5 & 2 & 2 needle flat seam elastic attach under band \\
\hline 6 & 2 & 3 step zigzag elastic attach back appex \\
\hline 7 & 2 & Bar Tack at front apex edge \\
\hline 8 & 3 & Hook and eye insert \& care label \\
\hline 9 & 3 & Trimming \\
\hline TOTAL & 19 & \\
\hline
\end{tabular}

\section{Case II - Balancing the Production Line for 450 Garments per Day Determination of the first workstation}

First, a feasible operation and one operator were added to the first workstation. Then, the work of the first workstation was simulated and at the end of the run, daily production output of the first workstation was analysed in terms of $\Theta$ value. The calculated value of $\Theta$ was 1. It implied that the workstation with one operator is in a position to achieve the required daily production target, 450 garments per day and it has the extra capacity to perform more operations. According to the precedence diagram (Figure 2), there are feasible operations that can be added to the first workstation. In this balancing process, maximum number of operations that can be added to one workstation is two. But, still only one operation was added to the first workstation. Therefore, another feasible operation was added to the first workstation in addition to the previous operation. After that, work of the first workstation was simulated and the daily production output of the first workstation was analysed by calculating $\Theta$. Calculated value of $\Theta$ was 1 . Therefore it can be concluded that the first workstation with one operator and two operations is in a position to achieve 450 garments per day. Furthermore, it was decided that the first workstation has the extra capacity to perform additional operations. According to the precedence diagram, there are feasible operations that can be added to the first workstation. But, when balancing this production line, more than two 
operations were not assigned to a single workstation. Consequently, it was concluded that one operator is adequate for the first workstation. He is able to perform two feasible operations so that the required daily production target is achieved.

\section{Determination of the second workstation}

After determining number of operators and feasible operations for the first workstation, a feasible operation and one operator were added to the second workstation. Then, the work of the first workstation and the second workstation was simulated, and at the end of the run, simulated daily production output was analysed in terms of $\Theta$ value. Calculated $\Theta$ value was 1. So, it was concluded that both workstations are in a position to achieve the required daily production target. In addition, it implied that the second workstation has the extra capacity to perform additional operations. According to the precedence diagram, there are feasible operations that can be added to the second workstation. In this balancing process, maximum number of operations that can be added to one workstation is two. But, still only one operation has been added to the second workstation. Therefore, another feasible operation was added to the second workstation in addition to the previous operation. After that, work of the both workstations was simulated and the daily production output of the second workstation was analysed by using $\Theta$. The calculated value of $\Theta$ was 1 . Accordingly, it can be decided that the second workstation has the extra capacity to perform additional operations in addition to the achievement of the daily production target. According to the precedence diagram, there are feasible operations that can be added to the second workstation. But, when balancing this production line, more than two operations cannot be assigned to a single workstation. Therefore, other feasible operations were not added to the workstation. Consequently, it was concluded that one operator is adequate to perform the assigned feasible operations of the second workstation in order to achieve the required target. Above procedure was applied to determine all workstations for the production line assuming the daily production target of 450 garments. The result of the analysis is shown in the table 3 . 
Table 3: Work allocations for 450 garments per day

\begin{tabular}{|c|c|l|}
\hline Workstation & $\begin{array}{c}\text { Number of } \\
\text { Operators }\end{array}$ & \multicolumn{1}{c|}{ Operation } \\
\hline \multirow{2}{*}{1} & 1 & Pre-fix centre front over lap \\
\cline { 3 - 3 } & \multirow{2}{*}{2} & Two needle join side seam \\
\hline \multirow{2}{*}{3} & 1 & Zig-Zag binding under arm \\
\cline { 3 - 3 } & \multirow{2}{*}{1} & 2 needle flat seam elastic cup neck edge \\
\cline { 3 - 3 } & \multirow{2}{*}{4} & 3 step zigzag elastic attach back appex \\
\hline 5 & 1 & Bar Tack at front apex edge \\
\cline { 3 - 4 } & 1 & Hook and eye insert \& care label \\
\hline 5 & $\mathbf{5}$ & \\
\hline Total & &
\end{tabular}

As seen in the table 3, five operators are required to achieve the daily production target of 450 garments per day.

\section{Value of the proposed algorithm}

The suggested line balancing procedure uses the simulation as a tool. It enables us to take in to account the reality. In this approach, standard minute values of operations are not taken in to consideration. Instead, the natural variations of processing times are modelled as probability distributions and those distributions are used for the analysis. It is closer to the reality. In the real production line, entities are moved as bundles. The bundle size is 48 . The impact of moving bundles through the line is not concerned in the current procedures. But, in the suggested approach, movement of bundles through the line is considered. Occasionally, operators waste a certain amount of time period to relax during their working time. Nevertheless, the influence of the above mentioned break down is not concerned in the current procedure. In contrast, the suggested method is strong enough to consider any kind of breakdown of any resource. In addition, every operator must count the number of garments in bundles before passing them to the next workstation to ensure the quantity. Also, operators spent a considerable time for bundle handling activities. In the current procedure, a certain percentage of allowance of the operating time is provided when calculating standard minute values. But, in the suggested approach, all above mentioned non-productive activities are taken in to consideration with variations. In 
summary, the suggested approach concerns the reality as compared to the existing balancing technique. The assignment of operators and operations are done in the proposed algorithm based on the expected output rate with the help of simulation. Therefore, we can conclude that the application of proposed algorithm to balance a production line reduces the gap between the expected production target and the real achievement.

\section{Recommendations and Future Research}

In the suggested line balancing approach, the SMV concept was not used. Instead, standard probability distributions were found and used for operating times. Developing a SPD (Standard Probability Distribution) database for all types of operations with regard to the garment manufacturing process is extremely important. These distributions reflect the real variations of processing times. Thus, managers can develop production plans which are closer to the reality. After developing a SPD database, it can be used to train newly recruited employees. Developing a training guide based on the SPD concept would be an important research so that it enables to facilitate trainers who are working in the production related areas. Due to various limitations, only the variation of operators' task times was concerned for this analysis. In addition, different types of disturbances such as machine breakdown, labour absenteeism, reworking, getting instructions from supervisors can be taken in to consideration to balance a production line. In this analysis, considered bundle size was forty eight (48), which is used in the production line. After balancing a production line one can determine the optimal bundle size which gives the maximum benefit to the company as well as operators. 


\section{References}

Baybars, I. (1986). A survey of exact algorithms for the simple assembly line balancing problem. Management Science, 32(8), 909-932.

Bhattacharjee, T.K., \& Sahu, S. (1987). A critique of some current assembly line balancing techniques. International Journal of Operations and Production Management, 7(6), 32-43.

Güner, M. G., \& Ünal, C. (2008).Line balancing in the apparel industry using simulation techniques. Fibres \& Textiles in Eastern Europe, 16(2), 75-78.

Konz, S., \& Johnson, S. (2004). Work design: Occupational ergonomics. Scottsdale: Holcomb Hathaway Publishers.

Kursun, S., \& Kalaoglu, F. (2009).Simulation of production line balancing in apparel manufacturing. Fibres \& Textiles in Eastern Europe, 17(4), 75.

Nkasu, M. M., \& Leung, K. H. (1995). A stochastic approach to assembly line balancing. The International Journal of Production Research, 33(4), 975-991.

Papadopoulos, C. T., O’Kelly, M. E., Vidalis, M. J., \& Spinellis, D. (2009). Analysis and design of discrete part production lines. New York: Springer. 